\title{
De Cartagena a Córdoba: balance y perspectivas de la educación superior en América Latina y el Caribe
}

\begin{abstract}
Axel Didriksson*
Resumen. En el contexto de un nuevo orden económico y político — con gobiernos de ultraderecha en Estados Unidos y países de Europa o Asia- y una creciente polarización social, la universidad pública enfrenta diversos problemas y cuestionamientos. El principal embate proviene del modelo económico neoliberal, que valora el conocimiento académico como una mercancía, y bajo esa lógica organiza la educación superior como un servicio global con fines de lucro. No obstante, se depliegan movimientos a favor de la legitimación y redefinición del quehacer universitario centrado en otros principios: ser un bien público y social, calidad con pertinencia, conocimiento a favor de un nuevo mundo con justicia, libertad y sostenibilidad. La autonomía y responsabilidad social universitaria, el presente y futuro cobran un nuevo sentido y están en el orden del día de los debates internacionales y regionales. La secuencia de crisis de onda corta generaron décadas de atraso en la región debido a la contracción de las responsabilidades gubernamentales en la educación y la proliferación de mecanismos de mercado para la regulación de la organización académica, así como a la ampliación de brechas en conocimiento, aprendizaje y desarrollo de la ciencia y la tecnología. Se formulan propuestas concretas para sustentar estrategias de cambio y transformar radicalmente a las universidades de la región.

Palabras clave: universidad, educación superior, crisis, transformación, América Latina.
\end{abstract}

* Investigador titular del Instituto de Investigaciones sobre la Universidad y la Educación (IISUE) de la Universidad Nacional Autónoma de México (UNAM), México. 


\section{From Cartagena to Cordoba: Balance and Perspectives in Higher Education in Latin America and the Caribbean}

Abstract. In a situation where social polarization and a new political and economic orden are imposed (by the ultra-right governments of the United States, Asia or Europe), the university is under scrutiny, in some cases excessively so from certain social sectors, though the brunt of the attacks emanate from the dominant neoliberal economic model, which values academic knowledge as a product, an within this logic organizes higher education as a profit-seeking global service, while simultaneously making a show of concern for the legitimization and redefinition of its mission based on other core principles: as a public or social good, quality and relevance, knowledge in favour of a new world with justice, liberty and sustainability. On this theme, the debates about the foundational characteristics of the university have been presented in various bodies, meetings and fora. The topics of university autonomy and social responsibility, the presnt and future of the public university, assume a new urgency and are the «order of the day» in international and regional discussions. The recent crisis is the result of decades of lag in the region, due to the contraction in the responsibilities for education by governments, the spread of market mechanisms for the regulation of academic organizations, and the propagation of gaps in knowledge, learning and the development of modern science and technology. Given these challenges, concrete proposals are offered in order to feed strategies for change in order to radically transform universities in the region.

Keywords: university, higher education, crisis, transformation, Latin America. 


\section{Antecedentes: el marco de acción de las CRES-UNESCO, 1996-2008}

En el mes de junio de 2018 se cumplieron 100 años de la publicación del Manifiesto Liminar de la Federación Universitaria de la Universidad de Córdoba, Argentina. Este evento estuvo acompañado de otro, la Conferencia Regional de Educación Superior (CRES) de la UNESCO, en el mismo sitio y en las mismas fechas.

Las conferencias regionales y mundiales de la UNESCO sobre educación superior han sido un referente esencial en la discusión sobre la universidad en la región y a nivel global. La primera regional, organizada en América Latina y el Caribe, ocurrió en la ciudad de La Habana, Cuba, en 1996, y la primera mundial en 1998, en la sede de ese organismo, en París. La segunda, masiva y contundente por la fuerza de la declaración de los universitarios latinoamericanos y caribeños, que se expresó en un texto que guarda enorme vigencia y actualidad, se celebró en la ciudad de Cartagena de Indias, Colombia, en 2008, y luego la mundial, también en París, en 2009. Entre ambas se organizó una reunión intermedia bajo el título de Conferencia +5 .

De ellas, debe destacarse que desde los resolutivos de la primera se logró transformar el otrora Centro Regional para la Educación Superior (Cresalc) en un instituto internacional de la UNESCO (el actual IESALC) y que además en el primer párrafo de la Declaración Mundial de 1998, celebrada en la sede de la UNESCO, se sustentara la idea de «la educación universitaria y superior como un bien social, un derecho humano y un beneficio para todos con el financiamiento del Estado, con autonomía y responsabilidad social y no como una mercancía ni tampoco como un servicio que conlleve montos de rentas de beneficio a particulares». 
Estos eventos fueron altamente significativos para los estudiosos del tema, para los representantes de las instituciones de educación superior en los niveles regional e internacional, y para los gobiernos miembros de la UNESCO, porque se organizaron en el contexto de los grandes cambios que están ocurriendo en la universidad, en el marco de un decisorio debate sobre la existencia, orientación, organización y compromiso social de la universidad en el actual siglo.

Lo que se presentó en las reuniones a las que se ha hecho referencia es fundamental para el debate que se presenta en la actualidad en la región, dada la profundidad que ha alcanzado la crisis de desigualdad económica-social y medioambiental a escala planetaria, las amenazas que se ciernen sobre enormes grupos humanos sumidos en la hambruna, la desesperación, la marginalidad y la expulsión de sus lugares de vida y de trabajo, y porque el entorno en el que éstas se celebran y organizan da cuenta de que todo se ha alterado y «diluido» (dixit, Bauman, 2017).

El cambio de toda una época tan lejana pero tan cercana está más que presente. La universidad está cuestionada y muy a menudo rebasada por algunos sectores de la sociedad, pero sobre todo por el modelo dominante de economía de corte neoliberal actual, que ha valorado el conocimiento académico como una mercancía y ha organizado la educación superior como un servicio global con fines de lucro, al tiempo que ocurren de forma paralela movimientos de legitimación y redefinición de su quehacer desde otra muy distinta perspectiva — desde su bien público y social, su calidad con pertinencia, los conocimientos a favor de un nuevo mundo con justicia, libertad y sostenibilidad—, en un contexto en el que la polarización social y un nuevo orden de imposición política y económica se están imponiendo (con los gobiernos de ultraderecha en Estados Unidos, Asia o Europa). 


\section{El debate sobre los fundamentos de la universidad}

En el marco de los acontecimientos mencionados de manera general con antelación, y por la discusión vigente, así como los temas de la autonomía y de la responsabilidad social universitaria, el presente y el futuro de la misma universidad pública cobra un nuevo sentido y está, y estará, en el centro del debate regional.

De mediados de los 1980 y hasta bien entrada la primera década del nuevo siglo, desde las denominadas metas del milenio, la «Educación para Todos», de la Organización de las Naciones Unidas (ONU) y de su organismo especializado, la Organización de las Naciones Unidas para la Educación, la Ciencia y la Cultura (UNESCO), o las estrategias de competitividad de algunos organismos multilaterales o internacionales, como la influyente Organización para la Cooperación y el Desarrollo Económicos (OCDE), la educación superior aparecía sólo como una prioridad de esfuerzos medios, porque estaba por abajo de alcanzar el objetivo de la universalización de la educación básica.

Esto cambió de forma radical a finales de los 1990 cuando empezaron a presentarse y debatirse sendos estudios respecto del impacto de la universidad y de las Instituciones de Educación Superior (IES) en el desarrollo económico, la emergencia de economías del conocimiento y, de particular importancia (sobre todo para este trabajo) de los resolutivos y los planes de acción de las reuniones regionales e internacionales sobre la educación superior de la UNESCO (véase Didriksson, 2007). En éstas, el papel de los resolutivos de la región de América Latina y el Caribe, desde muchas perspectivas, fue definitivo.

El debate que tuvo lugar en 2008 posicionó y propició a nivel mundial el desarrollo de dos paradigmas — contrapuestos — sobre el sentido y la 
orientación de la educación superior — que siguen vigentes, desde entonces, a nivel global. El posicionamiento de parte del Grupo Latinoamericano y Caribeño (conocido como Grulac) en las reuniones mencionadas logró postular los principios de vigencia de la universidad pública, a pesar de la presión de los países más ricos, de la OCDE y de muchos gobiernos de diversas partes del mundo - inclusive de países no desarrollados de Asia o África- que sostuvieron el enfoque de una educación superior como servicio, como bien negociable y como una jugosa parte del mercado que genera millonarias ganancias a sus propietarios. ${ }^{1}$

Como producto de la Segunda Reunión Regional, el documento suscrito de forma masiva, y que se daría a conocer también en la reunión mundial de la UNESCO durante el año 2009, generó uno de los posicionamientos más claros y definidos respecto del paradigma de bien social y de responsabilidad universitaria que se conozcan y que sigue vigente a pesar de que ha pasado una década desde entonces.

En este documento se postuló una concepción de educación superior como bien público y como instrumento estratégico de desarrollo sustentable, de cooperación internacional desde prácticas de integración regional, ${ }^{2}$

${ }^{1}$ Los momentos cruciales de este debate y sus diferencias políticas y conceptuales han sido profusamente documentados (cfr. Días, 2016; Didriksson, 2007).

${ }^{2}$ En esta Declaración Final se dice: «La educación superior es un bien público social, un derecho humano universal y un deber del Estado. Esta es la convicción y la base para el papel estratégico que debe cumplir en los procesos de desarrollo sustentable de los países de la región (...) Y se agrega la idea de un escenario que refuerce el compromiso social de la educación superior, su calidad y pertinencia y la autonomía de las instituciones (...) teniendo como meta el logro de una mayor cobertura social con calidad, equidad y compromiso con nuestros pueblos (...) (con el fin de impulsar) alternativas e innovaciones en las propuestas educativas. Asimismo, se incorporan las ideas de interculturalidad, producción y transferencia de conocimientos (...) para edificar una sociedad latinoamericana y caribeña diversa, fuerte, solidaria y perfectamente integrada» (UNESCO, 2008:10). 
buscando la conformación de un Espacio Común del Conocimiento y de Educación Superior en América Latina y el Caribe. Estos conceptos fueron detallados y desarrollados en su Declaración Final y en su Plan de Acción.

En estos documentos se plantea: a) Impulsar la expansión de la cobertura en educación superior, tanto en pregrado como en posgrado, con calidad, pertinencia e inclusión social. b) Promover políticas de acreditación, evaluación y aseguramiento de la calidad. c) Fomentar la innovación educativa y la investigación en todos los niveles. d) Construir una agenda regional de Ciencia, Tecnología e Innovación para la superación de brechas, con vistas a un desarrollo sustentable en la región, acorde a las políticas generales de cada Estado miembro (de la UNESCO). e) Reivindicar la necesaria transformación de las universidades, de la enseñanza y los aprendizajes, así como la plena vigencia de la formación humanística e integral de las personas.f) Propugnar por la integración regional latinoamericana y caribeña y la internacionalización de la educación superior mediante, entre otras iniciativas, la construcción del Espacio de Encuentro Latinoamericano y Caribeño de Educación Superior (Enlaces) como parte de la agenda de los gobiernos, de los organismos multilaterales y de las mismas universidades.g) Asumir una abierta y clara posición antimercantilista en la educación superior, donde se procure una muy estricta regulación sobre los servicios educativos de los proveedores internacionales.

Además, se destacan cinco prioridades: 1. Expansión de la cobertura de la educación superior, con calidad e inclusión social. 2. Políticas de acreditación, evaluación y aseguramiento de la calidad. 3. Promover la innovación educativa. 4. Construir una agenda de ciencia y tecnología para la superación de brechas y para el desarrollo sostenible de la región. 5. Promover la integración regional y la internacionalización de la educación superior, sobre todo con la construcción de Enlaces. 
Estos documentos fueron suscritos de manera muy amplia en la Reunión Regional de 2008 y, como se ha indicado, fueron ampliamente reconocidos en las declaraciones mundiales de la UNESCO, no así por parte de muchos gobiernos ni de otros tantos organismos transnacionales.

Para quienes suscribieron las declaraciones de referencia, sus términos y contenido fueron formalmente retomados y en algunos también pasaron a formar parte de sus definiciones de política pública. Para otros tantos, los resolutivos del evento mundial fueron considerados de forma muy crítica en contra de la UNESCO, y se dio por sentado que de ninguna manera esto constituiría un compromiso alguno, sino por el contrario: el impulso de políticas que deberían de orientar la educación superior como un servicio global, a condicionar el financiamiento público hacia éstas y promover un modelo de mercantilización, que en muchos países se ha definido como la descorporativización de las universidades públicas y privadas hacia modelos de «clase mundial» (World Class University), desde estándares de competitividad establecidos por los distintos rankings mundiales. ${ }^{3}$

\section{La transición}

A partir de la secuencia de crisis políticas y económicas cíclicas que se han vivido durante este siglo, la profundización de la pobreza, las inhumanas migraciones masivas y la extensión de los niveles globales de desigualdad, la Organización de las Naciones Unidas (ONU) tuvo que reconocer el fracaso de sus denominadas «Metas del Milenio», al igual que la UNESCO respecto de su

${ }^{3}$ Véanse, por ejemplo, Cheol y Kehm (2013); Altramn y Ebersberg (2013); Temple (2015). 
programa prioritario de «Educación para Todos». Entre 2005 y 2010 las condiciones educativas en el mundo, más allá de lo alcanzado en los países más desarrollados y en algunas potencias emergentes (sobre todo de Asia Pacífico) podrían calificarse como fallidas, por múltiples razones, entre otras por la falta de compromisos políticos de muchos gobiernos signatarios de estos programas de convergencia multilateral.

Durante los años 2014 y 2015 fueron redefinidos estos programas y ahora se ha propuesto, como es ampliamente conocido, una nueva agenda en donde se plantean 17 metas interrelacionadas para el logro de un desarrollo sustentable hacia el 2030. El tema 4 de los Objetivos de Desarrollo Sostenible (ODS) hace referencia a la educación y, en éstos, por primera vez, se establece que el papel de la educación superior es crucial y estratégico para el logro del conjunto de las metas.

En términos generales, en los ODS se retoman de manera muy amplia los resolutivos de las conferencias mundiales sobre educación superior que fueron, como se ha mencionado, defendidas por los universitarios de América Latina y el Caribe, entre los que resaltan: la educación superior como un bien común y un derecho humano universal; lograr su gratuidad plena; la autonomía universitaria; las responsabilidad de las IES en la solución de los más importantes y trascendentes problemas de la sociedad, por la vía de la producción de un conocimiento responsable y de innovación hacia el bienestar de la sociedad; y el compromiso para erradicar los males que se han reproducido en este siglo de forma absolutamente alarmante. ${ }^{4}$

${ }^{4}$ Debe subrayarse que con todo y esta definición la UNESCO ha publicado un documento reciente en el que presenta su ubicación desde el concepto de «bien público global» (cfr. UNESCO, 2015). 


\section{AXEL DIDRIKSSON}

En relación con la educación superior, la propuesta general de los ODS para 2030 es asegurar un amplio ingreso de la mayoría de la población del grupo de edad correspondiente, con calidad, equidad y plena inclusividad, desde plataformas de aprendizaje para toda la vida, para todos, con la responsabilidad social de la investigación y producción del conocimiento de las universidades para alcanzar un desarrollo sostenible.

Lo anterior tiene que ver directamente con las funciones sociales que llevan a cabo estas instituciones en el contexto del actual periodo y ante sus enormes desafíos: cambios en la segmentación de los mercados laborales, avances tecnológicos, urbanización, migración, inestabilidad política, degradación ambiental, desastres naturales, competencia por los recursos naturales, cambios demográficos, incremento del desempleo global, persistencia de la pobreza, ampliación de los niveles de inseguridad y amenazas a la paz y la salvaguarda de las personas, en particular, de manera magnificada, en el contexto de los países menos desarrollados y de algunas de las denominadas economías emergentes.

Este papel de la educación superior en el logro de un desarrollo sustentable plantea, sin embargo, un debate que debe diferenciar el carácter más bien general, retórico y sobre todo formal, de sustentación de las metas propuestas, dado que en la realidad éstas no podrán cumplirse de manera sostenida (por lo menos para la mayoría de los países en desarrollo, que son la prioridad marcada por la ONU para ser alcanzadas en el año 2030), tal y como ya se está documentando en los estudios de seguimiento que realiza la misma UNESCO, en sus Informes de Seguimiento de la Educación (GEM, por sus siglas en inglés), propuestos y definidos en la Declaración de Incheon (septiembre de 2015), desde donde se está monitoreando, anualmente, el desempeño de los objetivos para la educación, así como su relación con el resto de los ODS. 
Con la diversidad desarticulada de los sistemas de educación superior en estos países, las metas planteadas en los ODS no podrán pues ser alcanzadas, si no ocurren cambios radicales en las universidades y en las IES, en sus plataformas orgánicas, en su visión de desarrollo social, en la orientación de la producción de sus conocimientos científico-tecnológicos, en su capacidad para innovar y en su curricula, desde definiciones estratégicas de responsabilidad social y de bien común; pero sobre todo si no se logran articular estrategias de acción compartidas con otros actores, gubernamentales y no-gubernamentales.

Así, por ejemplo, en el Reporte de Seguimiento de la UNESCO (GEM Report, 2016) se enfatiza que de no ocurrir cambios reales en las actuales tendencias de desarrollo durante los próximos cinco años será improbable, en la mayoría de los países en desarrollo, que puedan alcanzarse las metas planteadas para el 2030, porque ello sólo podría lograrse hasta el 2054 (de acuerdo con sus análisis de tipo proyectivo) $u$ otras hasta el 2080, en concreto las referidas al amplio acceso a la educación secundaria superior y a la educación superior; esto será casi imposible si continúan las tendencias de marginalización de los grupos indígenas, de las personas que viven en las zonas rurales o de los jóvenes en las zonas pobres de las grandes y medianas ciudades.

Para la region de América Latina y el Caribe, durante las últimas dos décadas, la brecha entre los jóvenes del grupo de edad de 18 a 24 años se ha ampliado o mantenido (dada la diversidad de países), pero no superado, con proporciones que van de 50 a 60 por ciento en las diferencias de oportunidades entre el quintil más alto y el más pobre; ello se presenta de forma más aguda en las poblaciones indígenas, rurales, en las marginadas de las ciudades, en las mujeres y en los adultos. 
Además, en la región se vive la contradicción de tener el más alto nivel de desigualdad social y estar en el primer lugar de crecimiento de la educación superior privada a nivel mundial (en una relación media de alrededor de 50 por ciento entre lo público y lo privado), lo cual agranda las brechas generacionales y las oportunidades de estudio entre los que pueden alcanzar una educación superior con estabilidad y logro, y los que no pueden siquiera acceder a ella o permanecer hasta la obtención de un grado académico.

En términos generales, en el monitoreo GEM de 2016 se encontró que la meta de alcanzar el acceso de 72.7 por ciento del grupo de edad en educación secundaria superior no podrá ser lograda en la región sino hasta 2080, si es que llega a presentarse una tendencia constante de expansión aceptable.

Sólo en algunos países, como en Cuba, Brasil, Ecuador, Venezuela, Argentina, Uruguay o Bolivia, se ha logrado impulsar una serie de políticas de aseguramiento de la calidad con equidad social y un mayor acceso a sectores poblacionales tradicionalmente marginados, pero no en la mayoría de los países de la región donde se sigue reproduciendo un currículum obsoleto, la misma organización fragmentada del conocimiento, el desarrollo de disciplinas estancas, recortes constantes en los presupuestos públicos, con una reducida capacidad para desarrollar una ciencia y tecnología de innovación social de amplio impacto.

En todos ellos las expresiones más claras de responsabilidad social y de autonomía se han mostrado, de forma constante, en especial en las manifestaciones de los estudiantes y también, ya sea acompañando estas manifestaciones o con demandas propias, de parte de grupos de profesores e investigadores. 


\section{Balance general. La universidad en América Latina y el Caribe: avances, retrasos y perspectivas}

Lo anterior es el reflejo de la secuencia de crisis de onda corta que condujo a varias décadas de atraso en la región, debido, entre otros factores, a la contracción de las responsabilidades de los gobiernos frente a la muy desigual problemática de la educación, tanto general como superior; a la proliferación de mecanismos de mercado para la regulación de la organización académica; y a la reproducción de las brechas en los conocimientos, los aprendizajes y los desarrollos de la ciencia y la tecnología modernas.

Así, desde esas tendencias generales, de fines del siglo XX y hacia la primera década del XXI, puede constatarse que las universidades públicas y las IES en general enfrentaron tendencias de cambio, como las siguientes:

a) Durante los años 1980 y 1990, las reformas en la educación superior tuvieron como eje la desregulación del Estado, la descentralización institucional y la fragmentación de los tipos y calidad de las instituciones, sobre todo por el incremento de la oferta privada y mercantil y los enfoques de atención economicistas en la demanda.

b) Se generalizaron mecanismos, organismos e instrumentos de evaluación, acreditación y control de los sistemas universitarios en todos los ámbitos posibles de sus tareas y actores: programas, sectores, personas e instituciones, dentro y fuera de las secretarías o ministerios de educación superior, con el propósito de influir en los niveles de calidad por la vía de la medición de sus productos y resultados.

c) Se hicieron presentes nuevos esquemas de internacionalización, influidos por los Acuerdos de Bolonia (el Proyecto Tuning, por ejemplo) que, 
sin embargo, alcanzaron un bajo nivel de impacto, ${ }^{5}$ junto con la expansión e influencia de «proveedores transnacionales» de programas académicos en línea y nuevos esquemas de financiamiento diversificado.

d) Se impulsaron cambios institucionales y en la gestión académica para favorecer segmentos y nichos de producción de conocimientos y de investigación orientada al mercado. No obstante, poco se avanzó en la puesta en marcha de reformas sustantivas en materia de organización de la investigación y el posgrado.

e) Se alcanzó una mayor presencia de la demanda que de la oferta educativa, lo que impactó de forma desfavorable a la universidad pública que se vio saturada, mientras crecía de forma moderada una oferta de institutos superiores de tipo técnico. Todo ello sin avanzar de forma importante en la superación de las brechas de desigualdad, desescolarización, deserción, abandono y marginación de los sectores tradicionalmente excluidos.

f) Recurrencia a impulsar reformas y decisiones tanto de corte administrativas y de gobierno, pero también hacia la creación de nuevas carreras y áreas de conocimiento, centros de investigación y desarrollo; impulso a publicaciones; crecimiento del número de programas de posgrado; y algunas «buenas prácticas» (siempre superadas por las «malas prácticas», por efecto de la reproducción de mecanismos burocráticos y políticos).

${ }^{5}$ «La comunicación del 2006, cumplir con la agenda de modernización de las universidades: educación, investigación e innovación, insiste en los mismos objetivos y sugiere nuevas medidas relacionadas con la formación, la investigación y la innovación para superar las deficiencias de las universidades europeas. En concreto, se recomienda a las universidades que asuman mayores cuotas de responsabilidad sobre su viabilidad financiera siendo más innovadoras, proactivas y diversificando las fuentes de financiación, estableciendo fuertes vínculos con la empresa y con la sociedad en general (...) Pasado el año 2010, podemos afirmar que la mayoría de los objetivos no se han conseguido» (Vidal y Vieira, 2014:19). 
g) Referencias selectas de instituciones que buscaron la implantación de modelos emergentes a nivel internacional, similares a los del Espacio Único de Educación Superior de la Unión Europea; asumiendo las recomendaciones derivadas de proyectos suscritos con organismos como la UNESCO, el Banco Mundial o la OCDE; o buscando impulsar modelos de «universidad de clase mundial» como las de los países del Asia Pacífico, Australia o países árabes.

h) Tendencias de cambio de fuerte componente nacional, como se expresó en los debates respecto a las leyes nacionales en Colombia, Argentina, Brasil, Ecuador, Venezuela, Costa Rica, Chile; o bien con iniciativas para avanzar en procesos subregionales de integración: Centroamérica (CSUCA), Caribe (UNICA), Cono Sur (AUGM), ALBA y, más recientemente, Unasur.

i) Sistemas de remuneraciones extraordinarias hacia los grupos académicos, como sistemas paralelos al desarrollo del escalafón institucional dados por emeritazgo, resultados de investigación o apoyo a la docencia.

Desde esas perspectivas, en el más reciente informe de CINDA, se comprueba que en la región se han mantenido, sin cambios sustanciales, los niveles de desigualdad en el acceso al nivel secundario y superior de educación:

En promedio en 2010, sólo 21.7 por ciento de los jóvenes de 20 a 24 años que pertenecían al quintil más pobre de sus respectivos países había terminado la educación secundaria (...) en contraste, 78.3 por ciento de sus pares del quintil más rico completaron este nivel de educación, es decir, una brecha de 56.6 puntos porcentuales separaba a ambos grupos, lo que significa que no hubo en este aspecto en la década pasada prácticamente ningún avance en 


\section{AXEL DIDRIKSSON}

la región, por cuanto en 2000 la misma brecha era de 57.3 puntos porcentuales (...). Para el caso del nivel superior, la realidad es más acuciante debido a que para 2013, la población con ese nivel educativo, alcanzó 46 por ciento en el quintil más rico, mientras que en el quintil más pobre fue de apenas 4 por ciento (CINDA, 2016:56 y 58).

Entre otros indicadores que muestran el pobre avance de la educación superior en la región, indicados en este informe, está el bajo nivel de producción científica en el uso y manejo de nuevas tecnologías, en la producción de investigación, desarrollo e innovación, en la escolaridad de adultos, en la importancia de este nivel en las políticas gubernamentales o bien en la incrementada oferta educativa de tipo privada.

Desde las anteriores perspectivas, las condiciones del desarrollo de un sector productor de conocimientos, de nuevos aprendizajes y oferta de nuevos perfiles institucionales, hasta finales del anterior siglo, se mantuvo aún constreñido y en muchos casos aparecía como secundario y marginado, con rezagos como los siguientes:

1. Una escasa legitimidad del quehacer científico, donde el conocimiento científico no está plenamente valorado ni auspiciado.

2. Una reducida plataforma de aprendizaje social, de tal manera que el desarrollo de habilidades, capacidades, destrezas, competencias y valores relacionados con la producción y transferencia de conocimientos no se promueve ni planifica, y su promoción se encuentra en condiciones de brechas abismales respecto de lo que ocurre en otras regiones del planeta.

3. Un escaso interés del sector productivo para desarrollar una capacidad endógena en ciencia y tecnología: 
Con las exportaciones concentradas en recursos naturales, y con mercados manufacturados relativamente pequeños y altamente protegidos, por mucho tiempo no existieron el estímulo o la necesidad de innovar. Nuestros estilos de vida y patrones de consumo trataron de imitar a los países industrializados. Siempre fue más fácil importar la tecnología «llave en mano». Aún después de la apertura comercial, las multinacionales adelantan casi todos sus programas de I\&D en los países desarrollados (Buendía, 1995:320).

Esta falta de coordinación, que no llega a expresarse en sistemas nacionales de innovación, aparece como uno de los más importantes obstáculos para alcanzar el desarrollo de un sector de producción de conocimientos.

4. Una falta de claridad en las estrategias de desarrollo científico, tecnológico y de educación superior. Con el retiro gradual del Estado en materia de financiamiento para la educación superior, la ciencia y la tecnología, se pensó que ello conllevaría un aumento de la oferta de inversión de parte del sector privado, lo que no ocurrió.

5. Una permanente fuga de cerebros, que drena el esfuerzo local y entrega recursos físicos y humanos para el desenvolvimiento de los conocimientos de otros países, pero no para que los mismos tengan una lógica de «retorno en reversa» y una adecuada transferencia de los mismos, con la organización de una relación de cooperación positiva hacia la superación de brechas, desequilibrios y asimetrías existentes.

La crítica al modelo «reproduccionista» (que en realidad ocultaba una crítica al modelo de cogobierno y autonomista), tenía como sustrato la argumentación de que el mismo mantenía una curricula obsoleta, una docencia repetitiva y superflua; o la idea de que el Estado no tenía porqué garantizar la totalidad de los recursos a las universidades públicas, sino 


\section{AXEL DIDRIKSSON}

propiciar la diversificación de la oferta privada, la inversión en nuevas tecnologías de la comunicación y la información que redefinirían los procesos de docencia y de organización tradicionales, creándose con ello una suerte de búsqueda de «modernidad», impulsada por la evaluación y acreditación de instituciones o programas, o por ser un referente en los rankings locales o internacionales.

Para las universidades e IES de la región, estos movimientos diversificaron y fragmentaron el modelo dominante de universidades de viejo corte «napoleónico» profesionalizante, pero el desafío más grande fue buscar caminos, como se ha señalado con antelación, de modernización por la vía de las agencias de acreditación y los consejos de evaluación. Si bien el caso chileno aparece en la perspectiva del análisis de los cambios organizacionales y de contenido de forma constante, durante el periodo actual, sobre todo por una suerte de «encubrimiento» de las diferencias entre lo público y lo privado, también en otros países el proceso de cambios estructurales empezó a cobrar notoriedad desde el debate sobre la «diferenciación» de los sistemas universitarios.

Sin embargo, en países donde no alcanzaron a definirse prioridades para impulsar cambios en las universidades o en los centros de innovación científica, o para el desarrollo de tecnologías de forma explícita y deliberada, o fueron otras las perspectivas de interés de los distintos gobiernos, o cuando la sociedad tampoco exige que esto pueda realizarse, ni los gobiernos locales están interesados ni conscientes del verdadero, prolongado y sistemático esfuerzo que se requiere para desplegar un modelo sustentado en la producción y transferencia de aprendizajes y conocimientos, lo único que pudo prefigurarse fue una inercia en la que aparecía el trabajo denodado, muchas veces marginal, de colectivos de académicos o de instituciones que 
alcanzaron a otorgar alguna importancia al destinar recursos a determinados proyectos de investigación o, a menudo, como parte de la tenacidad de grupos o individuos con una mayor visión y altura.

Cuando el Estado no cuenta ni le interesa tener una visión estructurada e innovadora para impulsar o intentar experimentar o promover la puesta en marcha de un modelo alternativo de educación, de ciencia y tecnología, lo que opera es un «dejar hacer y hacer pasar» favorable a la comercialización y privatización de las escuelas y universidades. En las condiciones de un país subdesarrollado, con una educación y una ciencia «marginales» a la vida social y económica del país, donde el desempleo o la fuga de talentos predominan, la baja inversión en investigación se reproduce con el tiempo y la educación superior sigue manteniéndose buena sólo para una élite, ello resulta fatal para poder alcanzar un nuevo desarrollo con justicia y equidad.

Es por lo anterior que durante lo que va del actual siglo la respuesta social se hizo presente, desde un emergente pronunciamiento de diversos actores que se manifestaron a favor de una gran transformación en las universidades, sean éstas públicas o privadas. Así, y para mencionar algunos casos, esto se presentó de manera multitudinaria en las demandas del movimiento estudiantil chileno (2011-2014), del puertorriqueño (2011-2012), del colombiano (2011) o del mexicano (2011-2012), que dan cuenta de un giro cualitativo concerniente a la manera como se habían presentado las exigencias de este sector respecto de los reglamentos y políticas, o de la tendencia dominante en el mundo académico de la agenda tradicional de debate sobre lo público y lo privado, con demostraciones que han trascendido el ámbito institucional hacia el político en los niveles nacional o subregional.

Desde otras perspectivas, en los casos de Argentina, Ecuador, Bolivia, Brasil, Venezuela o Uruguay, e incluso en el conglomerado centroamericano 
y caribeño, la discusión respecto de una nueva agenda para el conjunto de la educación superior también ha cobrado un enorme interés en las comunidades universitarias, y aún más allá en el ámbito de otros sectores de la sociedad y de la vida política nacional. Es el caso, por ejemplo, de Ecuador, cuando se aprobó la Ley Orgánica de Educación Superior (LOES) (2010) bajo una fuerte movilización universitaria, pero que ha traído consigo la redefinición de conjunto de la política pública hacia la educación superior en ese país; o en Brasil, donde se han ensayado y puesto en marcha sendos programas afirmativos hacia las minorías y sectores de tradicional marginalidad, y su importante avance en materia de posgraduación (sobre todo al nivel de doctorado) y de investigación científica. Para mayor referencia, también están los caminos opuestos que se han presentado en Chile y Argentina, que dan cuenta de casos harto diferenciados en el tratamiento del significado del bien público y de la responsabilidad del Estado frente al interés público y privado. Otro tanto se ha presentado en Colombia, en Bolivia, en Perú o en Costa Rica, tal y como se da cuenta más adelante.

En términos generales, el balance de toda una época es que en la región se sigue manteniendo un sistema abigarrado pero desestructurado, diverso pero no complementario, autónomo pero no cooperante, cada vez más complejo pero no por ello más activo y actuante en el desarrollo de una sociedad del conocimiento sustentable y de bienestar para todos.

En este marco de tendencias y cambios, de fracturas y desigualdades, la idea tradicional de una universidad de modelo hegemónico público, con el énfasis en su responsabilidad de tipo «profesionalizante», con la idea de que su principal tarea consiste en ampliar y garantizar el más amplio acceso a este nivel educativo, y con la referencia a esquemas de gestión y organización basados en el cogobierno, la autonomía institucional, el subsidio 
del Estado y el dominio de una curricula de corte liberal y disciplinaria, empezó a dejar de ser el «modelo» predominante en la región.

Y, isin embargo se mueve!..., porque desde hace un par de décadas empezó la era de las instituciones universitarias emergentes, la creación de sedes y subsedes de universidades muy importantes y la constitución de otras tantas «nuevas» de factura innovadora o «emprendedora», pero también de una creciente ola de privatización y mercantilización.

Entre estas experiencias y reformas se están presentando esquemas de innovación académica, conceptos, políticas y programas que permiten constatar una suerte de nueva ola de cambios en la educación superior en la región, que tienen como sustrato el debate sobre lo que aquí se denomina una nueva agenda de transformación, ${ }^{6}$ muy relacionado con la Declaración de Cartagena de 2008, tal y como se ha mencionado con antelación, en la perspectiva de la participación de las universidades en el contexto de lo que está ocurriendo en el carácter y orientación de sus gobiernos.

Los ejemplos son afortunadamente múltiples y en muchos casos emblemáticos, porque representan el esfuerzo por trascender los modelos tradicionales de universidad, desde opciones distintas. Se mencionan, dado el espacio de este trabajo, algunos de los más conocidos (en orden alfabético): ${ }^{7}$

Argentina. Quizá se trate del país que más interés ha concentrado en la creación de nuevas universidades públicas, con subsidio del Estado, y que más ha defendido y legislado alrededor del concepto de bien público. De ello da cuenta que, en tan sólo algunos años, se han creado nueve universidades nacionales, tanto dentro como fuera del perímetro del «Gran Buenos Aires»,

${ }^{6}$ Véase, Didriksson (2012).

${ }^{7}$ Para la revisión de los estudios de caso que se mencionan véase Didriksson y Moreno (2016). 
donde habría que incluir la expansión de subsedes de la emblemática Universidad de Buenos Aires (UBA), con 12 centros regionales (en zonas del alta marginación), y las restantes al interior del país, que bien vale destacar que están prefigurando un esquema de descentralización novedosa, sobre todo en las provincias de Córdoba, San Luis, Entre Ríos, entre otras.

Brasil. Se están impulsando 18 nuevas universidades e IES bajo un esquema de innovación y responsabilidad de Estado: se hace referencia a la Universidad para la Integración Latinoamericana (Unila) y la Universidad Afro-Brasileña, de estructuras y ofertas académicas y de orientación estratégica para este país, fundamentales para trastocar la visión de la idea de universidad tradicional que trasciende sus mismas referencias. Asimismo, a partir de 2011 y bajo el gobierno del presidente Ignacio (Lula) da Silva, el incremento de universidades públicas fue sobresaliente (60), con énfasis en la creación de instituciones de tipo estatal, sin demérito de las de tipo federal, como las que se han mencionado. Importante el dato, cuando se conoce que en este país existía un tradicional predominio de la oferta privada frente a la pública.

Colombia. También con el objetivo de ampliar los niveles de cobertura, en un país de alta concentración de la oferta universitaria privada, se han impulsado Centros Regionales de Educación Superior (Ceres), con una modalidad público-privada de organización y financiamiento, así como bajo modelos híbridos que combinan lo presencial con la educación virtual, ubicados en localidades de poca cobertura de las IES tradicionales o de las grandes universidades privadas. Para 2012, se contaba ya con 176 de estos centros, con más de 30 mil estudiantes. Desde 2014, Colombia deja de tener un sistema de educación superior predominantemente privado y ahora el sector público es un poco mayor a 50 por ciento del tamaño de la matrícula. 
Ecuador. A raíz de la promulgación de la LOES se han venido impulsando, desde la Secretaría Nacional de Educación Superior, Ciencia y Tecnología (Senescyt), importantes cambios en el sistema de educación superior. Destaca la creación de cuatro nuevas universidades, denominadas «emblemáticas», como lo son la Universidad Nacional de Educación (Unae), la Universidad Regional Amazónica (IKIAM), la Universidad de las Artes (Uniartes) y la Universidad de Investigación Experimental Tecnológica (YACHAY), ya en marcha, de carácter público, con la perspectiva de impulsar un modelo de transformación, tal y como se ha planteado desde su ministerio rector: «En Ecuador, a partir de 2008, el Gobierno empieza públicamente a interpelar crítica y propositivamente a la universidad ecuatoriana, iniciando con esto un proceso de transformación inédito en el sector de la educación superior desde el retorno a la democracia en 1979» (dixit, René Ramírez, Secretario de Educación Superior, Ciencia y Tecnología de Ecuador, 2010).

México. Con todo y que desde hace décadas el Estado mexicano no ha contribuido a la creación de nuevas universidades federales, si se han constituido una serie de instituciones de financiamiento «dual», federal-estatal: la Universidad Autónoma de la Ciudad de México y la Universidad de la Ciénega de Michoacán, como modelos alternativos, fomentados y auspiciados por sus respectivos gobiernos locales. A nivel de política pública, los dos últimos gobiernos mexicanos han impulsado la creación de IES a nivel estatal, de tipo intermedio y de tipo «híbrido» en su financiamiento (federal-estatal-privado), denominadas como universidades tecnológicas, politécnicas, institutos tecnológicos estatales o interculturales. Asimismo, las universidades federales y autónomas más importantes han impulsado la creación de sedes alternas, también estatales, como subcampus o extensiones de las 
mismas, como la UNAM, la UAM, el IPN, la Universidad de Guadalajara, u otras. Vale destacar la creación, en este abigarrado enjambre, de la Universidad Nacional Abierta y a Distancia, que está en proceso de reestructuración para ampliar sus niveles de cobertura y calidad. En perspectiva, hacia el 2018 se pretendía crear 69 universidades de tipo intermedio (técnico), 30 nuevos campus como extensión de las universidades consolidadas y cuatro universidades federales (meta ya incumplida, sin embargo), aunque aún no se ve, por el momento, bajo qué modelo serán organizadas. Se prevé, sin embargo, como ya ha ocurrido en el sistema de creación de las instituciones intermedias referidas, que sus modelos de financiamiento sean mixtos, con fondos públicos como privados.

Paraguay. En este país se contaba tradicionalmente con una sola universidad. Para finales del pasado siglo ya existían siete universidades más ubicadas en el interior del mismo, sobre todo para dar respuesta al incremento de la demanda por estudios superiores, con lo cual también se presentó un crecimiento notable de la oferta privada por encima del crecimiento tendencial, como ha ocurrido en otros países de la región.

Perú. Desde inicios del presente siglo se han creado 21 universidades públicas en el interior de este país, pero también el crecimiento de la oferta privada ha sido constante. En el 2012 se decretó una moratoria para suspender el crecimiento de instituciones públicas, con el objetivo de replantear las políticas en el sector y redefinir el marco normativo de una nueva etapa.

Uruguay. Así como en Paraguay, en República Dominicana u otros países del Caribe o de Centroamérica, en este país se vivió durante décadas la existencia de una sola universidad asumida como el baluarte de la educación superior y la definitoria de las políticas sectoriales, como es el caso de la emblemática Universidad de la República. Con el nuevo siglo se 
creó una nueva institución: la Universidad Tecnológica del Uruguay (2013), y está en discusión la creación de una nueva universidad pública donde, junto con Argentina y Cuba, existe un fuerte predominio de lo público frente a lo privado.

Venezuela. El Gobierno Bolivariano de Venezuela, dentro de una gran controversia a nivel universitario respecto de la relación calidad-cantidad, se propuso impulsar una amplia regionalización y creación de sedes universitarias y no-universitarias, de tal manera que para inicios del presente siglo se contaba con 232 sedes y extensiones de IES, de las cuales 59 se ubicaban en la zona urbana de la ciudad de Caracas. No obstante, la matrícula de IES privadas alcanzó 77 por ciento de su total. Para ampliar los niveles de cobertura, se crearon las Aldeas Universitarias, las Universidades Politécnicas Territoriales y 20 nuevas universidades a lo largo y ancho del país de carácter público, dentro de una estrategia que se ha concentrado en la «universalización» de la tasa bruta de escolarización (TBE). De entre las nuevas universidades vale destacar la creación de la Universidad Bolivariana de Venezuela, la Universidad del Cine y la Universidad de las Fuerzas Armadas, que para 2010 ya habían alcanzado una matrícula de cerca de medio millón de estudiantes. Entre 2012 y 2013 se han creado cinco universidades estatales más, y para 2014 se planeaba crear cuatro nuevas universidades territoriales, en otros estados del país.

Este panorama general de cambio en algunos casos nacionales importantes -incompleto, sin ninguna duda, porque habría que incluir las tres universidades que están en desarrollo en Bolivia (Unibol), o muchas de las nuevas sedes de universidades nacionales, o los proyectos de articulación integracionistas que están prefigurando un nuevo escenario, por ejemplo, desde lo que se impulsa ahora por la AUGM o la Unasur (por mencionar 
este par de impresionantes esfuerzos multinacionales, en materia de educación superior, ciencia y tecnología) da cuenta de que en la región se está ingresando a un nuevo periodo de construcción institucional y de innovación académica y social, en el que empieza a destacar la constitución de plataformas de conocimientos y aprendizajes, uso y manejo extenso de nuevas tecnologías y la gestión de procesos de innovación, para ir empatando la idea de una «sociedad latinoamericana del conocimiento» o de un «espacio común del conocimiento», en la perspectiva de un énfasis en el bien social del valor de los estudios y la investigación universitaria.

En este debate, pues, se han presentado importantes acontecimientos universitarios y nuevas conformaciones institucionales, porque a pesar de las terribles circunstancias que se ciernen sobre nuestros países, en los casos que aquí se abordan se están impulsando cambios e innovaciones relevantes de creación de nuevas IES, con un renovado interés en ampliar y cualificar la base de investigadores, de producción endógena de conocimientos y sus relaciones con sistemas de innovación científico-tecnológica en una perspectiva integracionista.

\section{La importancia del debate en Córdoba 2018: prioridades para los próximos 10 años}

El proceso y la discusión generados en la reunión de París sobre educación superior (cfr. UNESCO, 2009) de parte del grupo de universitarios de América Latina y el Caribe, que buscó sustentar el término «bien público y social» para caracterizar la educación superior, son casi imperceptibles a la luz de lo que se ha discutido en este trabajo, a escala mundial, pero es para la región 
extremadamente importante. Simple y sencillamente porque busca hacer girar la balanza de los términos y de las prácticas hacia el lado del bien común y los derechos humanos universales, y desde la perspectiva de la autonomía tan relevante como lo es ahora.

Frente a la comercialización y mercantilización de la educación superior que se vive en distintas partes del mundo, y que ha calado hondo en la región de América Latina y el Caribe, la expresión de un «bien común» ayuda mucho a entender las nuevas perspectivas que se presentan para la autonomía de las universidades y sus límites, como garante de la preservación de los conocimientos y los aprendizajes con un sentido abierto, gratuito, socialmente amplio y comprometido con un desarrollo sustentable y equitativo, justo y democrático.

Desde el debate de la UNESCO de los años de referencia, dos conceptos relacionados al de la autonomía fueron los de «pertinencia» y de «responsabilidad social». A menudo se les ve de manera equivalentes, o se les trabaja de forma indiferenciada, pero vale la pena precisar su dimensión específica.

La pertinencia se relaciona con el contenido del trabajo académico, con el proceso a través del cual se determinan y seleccionan de forma consecutiva contenidos, métodos, lenguajes, se organizan los aprendizajes en aulas y laboratorios y se perfilan líneas y proyectos de investigación de los profesores o los investigadores. La pertinencia hace referencia a los procesos, avances y productos «internos» de la producción y transferencia de conocimientos de la universidad.

La responsabilidad social supone que la institución universitaria o de educación superior produce sus conocimientos y sus egresados con un perfil ético y ciudadano hacia afuera, hacia la sociedad, hacia los compromisos, posicionamientos, definiciones, como tareas que promueve desde 


\section{AXEL DIDRIKSSON}

sus funciones de docencia, investigación y difusión de la cultura hacia la sociedad en su conjunto, donde se hace notable la pertinencia del trabajo académico realizado desde su autonomía a favor del desarrollo y bienestar, de la educación y la cultura de una sociedad determinada.

Es esta diferenciación la que nos permite identificar las relaciones dinámicas entre la autonomía universitaria, la pertinencia del trabajo académico y la responsabilidad social en las resoluciones y avances de las universidades de América Latina y el Caribe, que tienen arraigo con instituciones autónomas, con historicidad y contundencia, con un particular modelo de universidad, distinto a los modelos «emprendedores», de «capitalismo académico» o híbridos de mercantilización y de innovación con fines de lucro.

Durante los años que van de 2006 a 2009, la región de América Latina y el Caribe impulsó un importante movimiento desde sus instituciones de educación superior, sobre todo desde sus universidades nacionales y públicas, de creación de redes y asociaciones regionales, subregionales y nacionales para definir que la reforma universitaria del siglo XXI debía estar férreamente enraizada en su autonomía, en la pertinencia de su trabajo académico y en la responsabilidad social de sus instituciones de educación superior y universidades, tanto públicas como privadas.

Las premisas fundamentales sobre las cuales la región se ha posicionado en el debate mundial, frente a las sociedades del conocimiento y sobre todo frente al desarrollo de economías del conocimiento, se concentran en la idea de mantener la educación superior (y la educación en general) como un bien público, en el rechazo a su comercialización y mercantilización, a la subordinación de las universidades y de sus institutos de investigación a las empresas y al mercado, y en la sustentación de universidades que promuevan el cambio con responsabilidad social. 
Desde estas premisas, se ha impulsado un muy importante debate en el seno de la UNESCO y otros organismos, sectores y universidades, en donde prevalece la controversia con el postulado impulsado por la OCDE de que la educación superior debe ser considerada como «un bien público global». Tanto en la reunión de Cartagena de la UNESCO de 2018, como en la también mencionada de París en 2009, los universitarios latinoamericanos se opusieron a la generalización de este término y postularon la educación como un bien social, a secas, como un derecho humano, lleno de contenido y de una autonomía responsable. En los términos de José Dias Sobrinho,

la educación superior no puede ser un «bien público global». Esta redefinición dentro de un plan global desvincula la educación de sus raíces históricas y de los medios concretos donde se insertan las instituciones y donde se producen los procesos formativos y los conocimientos. De esta manera, ello contradecía el sentido público de la educación en cuanto proceso primordial e insustituible para el desarrollo individual y social, la realización de los objetivos comunes de consolidación de las identidades culturales y la elevación de las condiciones de vida de los pueblos, en especial de países subdesarrollados (2008:94).

Las diferencias llegan a ser abismales en los procesos de cambios que ocurren en las universidades que participan directamente de los desarrollos de economías del conocimiento. En los países en los que esto ocurre, la inversión hacia la investigación fundamental, la experimentación científica, las patentes, los modelos de simulación de sistemas de inteligencia artificial, la infraestructura, el número de investigadores en activo y los 
contratos con fines de comercialización de los productos de investigación y de innovación tecnológica son una constante, y son parte de la vida de las universidades y de otros núcleos de producción de conocimientos. Ya se ha hecho referencia de ello bajo el término de «capitalismo académico», aunque no sólo en el sentido de una subordinación a los procesos de inversión y obtención de ganancias, sino también a los recientes fenómenos de transformación institucional para adecuar a las universidades a una relación simbiótica con las empresas que dependen de la generación de conocimientos y tecnologías.

En América Latina y el Caribe la realidad fue y es harto distinta. Son las universidades mayoritariamente públicas las que concentran la gran mayoría de la investigación fundamental y aplicada, son las que orientan la mayor inversión hacia investigación y desarrollo, las que forman los profesionales e investigadores que administran, reproducen u organizan la producción y transferencia de conocimientos y tecnologías, con todo y que las relaciones con las empresas sean aún minoritarias y hasta conflictivas; pero son, por encima de todo, la sede de la «inteligencia nacional», de lo mejor de los valores académicos, humanísticos y científicos de cada país, de su cultura y de su identidad.

Lo anterior ha propiciado el impulso de prácticas y procesos de convergencia, cooperación e intercambio, a escalas regional o internacional, desde perspectivas de cambio universitario que enfatizan la necesaria pertinencia, autonomía y responsabilidad social que tienen las instituciones de educación superior en general, como instituciones culturales, educativas, científicas, con intereses, proyectos y definiciones de futuro distintas a las de las empresas o los gobiernos. Se trata de un papel social desempeñado desde espacios únicos e indivisibles, que incluso han 
sido consagrados históricamente como «autónomos» — de allí su fuerza y su originalidad-, como un conglomerado diverso con una estructura, funciones, decisiones, organizaciones y personas, que trabajan desde bases propias y se sustentan cada vez más como un poder por sí mismo en la sociedad y también como parte del Estado.

De allí la importancia de redefinir, de nuevo, la autonomía universitaria, porque es desde esta posición que debe pensarse a sí misma de forma crítica e impulsar cambios fundamentales desde los nuevos paradigmas pedagógicos, epistémicos, científicos y tecnológicos, el desarrollo económico, la sustentabilidad, la interculturalidad y el futuro de la sociedad en la que se encuentra, cuando se pueden reconocer los enormes y agudos problemas de pobreza, inequidad, desigualdad, destrucción medioambiental y elevación del nivel de los conflictos sociales hacia los que puede contribuir a plantear soluciones factibles, inteligentes y responsables. En primerísimo lugar lo debería de hacer hacia la solución de la aguda problemática de desigualdad del sistema educativo, visto en su conjunto.

La autonomía universitaria, entonces, debe ser el pivote, la fuerza propulsora de cambios hacia el interior (por su pertinencia) y hacia la acción decidida de las instituciones de educación superior hacia afuera (por su responsabilidad social). En este sentido, la acción autónoma de las universidades no debe limitarse a un país determinado. Por lo menos en el contexto de la región de América Latina y el Caribe la cooperación, la organización de redes y asociaciones, de programas conjuntos, de esquemas originales de movilidad de estudiantes y académicos, es un proceso en marcha y esto está propiciando perspectivas estratégicas muy importantes para el desarrollo de una capacidad endógena de producción y transferencia de conocimientos (Didriksson, 2007). 
Así, el contenido de un «Nuevo Manifiesto» para la universidad de América Latina y el Caribe del siglo XXI debería de decir casi más o menos lo mismo que el de hace 100 años — si esto en verdad ocurriera en alguna universidad o en muchas relacionadas entre sí-, para proclamar una nueva hora para la integración latinoamericana, porque como afirma Pablo Gentili, «no se trata de repetir las palabras de orden, las banderas y propuestas de la Reforma de Córdoba, pero sí (...) de reconocer en la radicalidad de ese movimiento, los aportes que el mismo nos ha legado y la necesidad de reformularlo en virtud de una especificidad histórica que actualiza esa herencia en el marco de una nueva coyuntura» (Novaes y Dagnino, 2013:79).

Ubicadas como instituciones de gran trascendencia para fines de desarrollo económico, cultural y social, las expectativas que se ciernen sobre los conglomerados académicos de producción y transferencia de conocimientos, en lo particular las redes y asociaciones de universidades a nivel regional —con fuertes vínculos internacionales-, están provocando cambios de fondo y severos posicionamientos ante las cíclicas crisis económicas y sociales, exigiendo la redefinición de políticas y planes y, sobre todo, mayores recursos para desempeñar con calidad y pertinencia su vital función de ser una de las instituciones sociales que mejor contribuyen al desarrollo de una sociedad del conocimiento relacionado con el bienestar de la mayoría de su población.

Durante este periodo están ocurriendo múltiples acontecimientos y modificaciones en su entorno y contexto que no se habían previsto con antelación. Las perspectivas que se habían presentado con planteamientos de pensadores de gran influencia que preveían una relación directa, y a la vez simple, de subordinación entre la universidad hacia la empresa, bajo 
la forma de una extrema comercialización, no ha ocurrido en la realidad, sino de forma tendencial en algunas instituciones notables, sin duda, pero no de forma generalizada.

En la realidad, el tema de la autonomía se ha mutado en un proceso más complejo, porque en lugar de que se estuviera avanzando a paso firme hacia una sociedad del conocimiento está ocurriendo una situación diferenciada, en la que la educación, los aprendizajes y lo más consagrado del pensamiento humano está polarizando a las sociedades, abriendo brechas enormes en sus niveles de desigualdad social, alterando de forma brutal las condiciones de vida de quienes cuentan con información y un valor agregado sustancial en conocimientos frente a los que no los tienen, poniendo en peligro el planeta y sus especies, modificando los patrones genéticos de sociedades enteras, alterando el contenido de sus alimentos; en otras palabras, haciendo realidad una sociedad en constante riesgo de destrucción total.

Para la región, una sociedad del conocimiento está muy lejos de las perspectivas futuras de las próximas dos o tres décadas del nuevo siglo. Lo que si se está imponiendo es una nueva forma de uso y manejo de la inteligencia humana, de los conocimientos y de la información que puede producir, sistematizar y transferir socialmente, desde lógicas de ganancia y competitividad extremas, una economía que valoriza la fuerza de trabajo humana en nuevas proporciones, que articula instituciones enteras y centros de producción de conocimientos para alcanzar nuevas tasas de ganancia y mayores niveles de especulación financiera para alterar la base de relaciones económicas, y sus mediaciones - el valor de uso del trabajo por el valor de cambio de los aprendizajes - en la constitución de un nuevo modo de producción: el modo de producción sustentado en los conocimientos, dependiente y de alto carácter consumista. 
Lo que aquí se sugiere, frente al debate que trae y lleva a la autonomía, a la libertad académica, a la pertinencia y a la responsabilidad social de las universidades, es que hay que ponerse ahora en algún lado. El lado en el que se ubica este trabajo es en el que se ha construido la autonomía relacionada con un pensamiento renovador, múltiple, diferenciado, rico en contenido y al mismo tiempo integral, histórico y muy enraizado en las universidades públicas latinoamericanas. Esta es nuestra postura y es esta la manera como se presentan y se sostienen las posibilidades de un cambio fundamental en las universidades que buscan y reclaman un espacio propio, que ya ha sido duradero y que siempre ha estado presente.

Así, con vistas a las propuestas que se están debatiendo respecto de lo que puede impulsarse desde la región, tomando en cuenta los resolutivos obtenidos por la CRES-2018, nuestra postura propone sustentar estrategias de cambio desde las siguientes diez propuestas:

1. Refrendar la Declaración de la CRES-2008 y el Plan de Acción propuesto en esa reunión; y discutir la postura de la UNESCO respecto a su concepto reduccionista de «bien público global».

2. Definir un marco de acción y planificación para ser evaluado por una comisión de seguimiento, nombrada en la CRES-2018 de manera ex profeso, y sustentado en un plan de acción a cargo de una comisión ejecutiva de Enlaces (nombrada también en la CRES-2018), con el acompañamiento y «paraguas» del IESALC-UNESCO. Debe tomarse en cuenta que el «gran vacío» que dejó la CRES-2008 fue no haber podido concretar una dirección y la constitución de un equipo de trabajo encargado de poner en marcha su plan de acción aprobado. Ahora es cuando esto debe hacerse de manera prioritaria. La forma como deberán ser elegidos los miembros de esta dirección y de sus cuerpos colegiados de seguimiento, estudios y 
acción, debe ser parte central de la discusión de la CRES-2018, así como su ubicación (junto con la de sus nodos), su rotación, su temporalidad, sus principios, reglas de operación, fondos y capacidad de decisión.

3. Discutir y asumir los avances logrados por los organismos de integración con experiencias importantes alcanzadas, como la AUGM, la Unasur o el Mercosur, retomar redes de trabajo que se encuentran en un estado de excepción pero que son en extremo importantes (como la Red de Macrouniversidaes Públicas o el Espacio Común Iberoamericano del Conocimiento) y las que están surgiendo, con el fin de establecer marcos de acción desde sus particulares definiciones e identidades.

4. Considerar establecer grupos interinstitucionales de investigación e innovación en áreas y temas de gran relevancia para la región: violencia y solución de conflictos, gobernabilidad, sustentabilidad, interculturalidad, agua y recursos naturales, nuevas tecnologías (robótica, nanotecnología, biotecnología, inteligencia artificial, etcétera), energía, estudios sobre la complejidad, pobreza, inequidad, desigualdad, modelos de desarrollo económicos, estudios de género, ruralidad y urbanismo, etcétera.

5. Generar propuestas prácticas para que se flexibilice la curricula profesional tradicional o la emergente, con el fin de ampliar la movilidad de estudiantes y académicos por toda la región, de tal manera que la integración sea una realidad desde la academia, la cultura y el intercambio de experiencias y vivencias.

6. Impulsar un fondo de financiamiento permanente (por lo menos para los próximos cinco años y renovable cada tiempo) para la operación de Enlaces y la operación y apoyo de proyectos comunitarios, integracionistas y de innovación académica, científica y tecnológica. 
7. Exigir de forma decidida el incremento del subsidio del Estado hacia las universidades públicas, hasta alcanzar 1.5 por ciento del PIB para el periodo de los próximos cinco años.

8. Impulsar una adecuada y urgente regulación de los servicios privados en la educación superior, definidos por las universidades públicas, bajo el criterio de bien común, de derecho universal, de amplio acceso y de calidad con pertinencia nacional y regional. De ninguna debe permitirse la existencia de instituciones con fines de lucro o de proveedores internacionales privados sin ningún tipo de control en nuestros países. Todos los recursos públicos de los gobiernos deben ser orientados en exclusividad hacia las universidades con régimen de autonomía, para el pleno desarrollo de sus funciones de enseñanza, aprendizaje, investigación y difusión de la cultura.

9. Defender la organización del conocimiento en todos sus ámbitos y áreas disciplinares e interdisciplinares; de ninguna manera se debe permitir priorizar una de ellas por encima de otras y mucho menos marginar en los presupuestos y en las políticas públicas la importancia de las humanidades y las ciencias sociales. La premisa es que no hay ninguna ciencia ni ninguna tecnología que no sea social o de bien público.

10. Convocar a la transformación radical de las universidades de la región, a crear nuevas universidades, a ampliar la diversidad de las IES, a propiciar enfoques interculturales y de amplio acceso, y a garantizar una calidad con pertinencia; y, sobre todo, impulsar un enfoque de responsabilidad social en la investigación científica y tecnológica. 


\section{Referencias}

Altramn, Andreas y Bernd Ebersberg (eds.) (2013), Universities in change, managing higher education institutions in the age of globalization, New York, Springer.

Alderete, Ana María (comp.) (2013), El Manifiesto Liminar. Legado y debates contemporáneos, Argentina, Universidad Nacional de Córdoba.

Brunner, José Joaquín (coord.) (2016), Educación superior en Iberoamérica, informe 2016, Chile, Centro Interuniversitario de Desarrollo.

Centro Interuniversitario de Desarrollo (CINDA) (2006), Educación superior en Iberoamérica, Informe 2016, Santiago, RIL.

Días, Marco Antonio (2016), «Desafíos de la Educación Superior a la luz de la Reforma de Córdoba», Revista Integración y Conocimiento, 2(5).

Dias Sobrinho, José (2008), «Evaluación de la Educación Superior en América Latina», en Ana Lúcia Gazzola y Axel Didriksson, Tendencias de la Educación Superior en América Latina y el Caribe, Venezuela, Instituto Internacional para la Educación Superior en América Latina y el Caribe-Organización de las Naciones Unidas para la Educación, la Ciencia y la Cultura.

Didriksson, Axel (2012), «La nueva agenda de transformación de la Educación Superior en América Latina», Perfiles Educativos, XXXIV(138), México, Instituto de Investigaciones sobre la Universidad y la Educación-Universidad Nacional Autónoma de México.

(2007), Universidad y sociedades del conocimiento, México, Organización de las Naciones Unidas para la Educación, la Ciencia y la Cultura.

Didriksson, Axel y Carlos Iván Moreno (2016), Construyendo e innovando. La universidad en América Latina: estudios de caso, México, Global University 


\section{AXEL DIDRIKSSON}

Network for Innovation/Universidad de Guadalajara/Universidad Autónoma del Estado de México.

Gazzola, Ana Lúcia y Axel Didriksson (2008), Tendencias de la Educación Superior en América Latina y el Caribe, Venezuela, Instituto Internacional para la Educación Superior en América Latina y el Caribe-Organización de las Naciones Unidas para la Educación, la Ciencia y la Cultura.

Gómez Buendía, Hernando (coord.) (1995), Educación: la agenda del siglo XXI, Colombia, Programa de las Naciones Unidas para el Desarrollo/TM Editores. Jung, Cheol Shin y Barbara Kehn (eds.) (2013), Institutionalization of world class university in global competition, Dordrecht, Springer.

Novaes, Henrique y Renato Dagnino (2013), «La Reforma de Córdoba: una lectura contemporánea», en Ana María Alderete (comp.), El Manifiesto Liminar. Legado y debates contemporáneos, Argentina, Universidad Nacional de Córdoba.

Organización de las Naciones Unidas para la Educación, la Ciencia y la Cultura (UNESCO) (2008), Declaración y Plan de Acción de la Conferencia Regional de Educación Superior en América Latina y el Caribe, CRES 2008, en https:// unesdoc.unesco.org/ark:/48223/pf0000181453 (2015), Rethinking education: towards a global common good?, París, UNESCO.

Temple, Paul (2015), Universities in the knowledge economy, higher education organization and global change, Londres, Routledge.

Vidal, Javier y María José Vieira (enero-marzo, 2014), «Gobierno, autonomía y toma de decisiones en la Universidad», Bordón, Revista de Pedagogía, 66(1). 